\title{
Numerical Simulation of Fluid Flow Around Circular Cylinder and Three Passive Controls to Reduce Drag Coefficient at $\mathrm{Re}=500$
}

\author{
Chairul Imron, Amirul Hakam, Basuki Widodo and Tri Yogi Yuwono
}

\begin{abstract}
Numerical experiments and simulations of fluid flow through the outer surface of a circular cylinder and three passive controls have been investigated to determine the proper configuration of three passive controls in reducing the drag coefficient. One of passive controls is placed in front of the cylinder with distance ratio $(S / D)=2.4$ and the other two passive controls are placed behind the cylinder with distance ratio $(T / D)=1.6,1.8$. The angle between two passive controls behind the cylinder are $\alpha=30^{\circ}, 60^{\circ}, 90^{\circ}, 120^{\circ}$. The Navier-Stokes equations for incompressible, viscous and unsteady fluid flows is solved based on SIMPLE (Semi-Implicit for Pressure-Linked Equations) algorithms and discretized using finite-difference method. The difference in $\alpha$ affects the reduction in the drag coefficient significantly. The best configuration of three passive controls design is one of passive controls put at the distance ratio $S / D=2.4, T / D=1.6$ and $\alpha=60^{\circ}$. This configuration can reduce the drag coefficient optimally to $21.2109 \%$.
\end{abstract}

Index Terms-Drag coefficient, numerical simulation, passive control.

\section{INTRODUCTION}

$\mathbf{N}$ UMERICAL experiments and simulations of fluid flow through the outer surface of a circular cylinder and passive controls have been investigated. Circular cylinders will receive a drag due to the passing fluid. Drag will affect the physical endurance of a circular cylinder. Passive control is placed around a circular cylinder to reduce the drag coefficient received by the circular cylinder.

Some shapes modification of circular cylinder can reduce the drag coefficient more efficiently such as D-shape cylinder that a front face of cylinder is cut off and I-shape cylinder that front and rear faces are cut off with different cylindrical cutting angles [1], [2], [3]. At the cutting angles $50^{\circ}-53^{\circ}$ and $R e>2.3 \times 10^{4}$, the wake area becomes smaller and the vortex region moves away. So, the drag coefficient received can be reduced optimally.

Moreover to modifying the shape and Reynolds number $(R e)$, the number of passive controls used and its configurations also affect the amount of reduction of the drag

Manuscript received July 3, 2019; accepted December 16, 2019.

C. Imron, A. Hakam and B. Widodo are with the Department of Mathematics, Institut Teknologi Sepuluh Nopember, Surabaya 60111, Indonesia E-mail: imron-its@matematika.is.ac.id

T.Y. Yuwono is with the Department of Mechanical Engineering, Institut Teknologi Sepuluh Nopember, Surabaya 60111, Indonesia.

We appreciate the head of research institute and community service of Institut Teknologi Sepuluh Nopember (LPPM ITS) Surabaya Indonesia, on behalf of the Ministry of Technology and Higher Education who gave us a research grant under contract No: 010/SP2H/LT/DRPM/IV/2017, April, 20th 2017 , in giving us an opportunity to disseminate our results in this paper. coefficient. To obtain the optimal position configuration, Imron et al. has conducted research using a single passive control placed in front of a circular cylinder or behind it in varying distances. Then, it followed by two passive controls experiments placed in front of the circular cylinder and behind it. Some of the two passive control configurations are vertical position of I-Shape cylinder placed in front of circular cylinder and horizontal position of I-Shape cylinder placed behind the circular cylinder [4], the form of passive control in front is the same as before, but passive control behind is elliptical [5] and circular cylinder with ratio of diameter is $d / D=0.125$ [6]. The other passive control configurations are two small cylindrical passive controls placed behind a circular cylinder [7], two passive controls in the form of plates arranged in parallel placed behind the cylinder [8], six small cylindrical passive controls are placed around a circular cylinder [9].

Based on the results of the reduction in drag coefficients using two passive controls in previous studies, the current study discusses the optimal configuration of three passive controls to reduce the drag coefficient. One of the three passive controls is placed in front of the circular cylinder and the other two passive controls are placed behind the circular cylinder. The angle between the center of two passive controls behind the cylinder from the center of main cylinder is $\alpha$. The idea is two passive controls behind the cylinder can reduce the wake area behind the circular cylinder. So, the drag can be reduced.

\section{Methodology}

\section{A. Flow Equation}

The mathematical equation for fluid flow is constructed based on momentum and continuity equations in the form of a non-dimensional Navier-stokes equation [10] as follows :

$$
\begin{gathered}
\frac{\partial \mathbf{u}}{\partial t}+\nabla \cdot \mathbf{u u}=-\nabla \mathbf{p}+\frac{1}{R e} \nabla^{2} \mathbf{u}, \\
\nabla \cdot \mathbf{u}=0,
\end{gathered}
$$

where $\mathbf{u}$ is a form of non-dimensional vector velocity flow on the coordinate axis $(x, y), \mathbf{p}$ is the non-dimensional form of the pressure component and $R e$ is the Reynolds number.

\section{B. Numerical Solution}

The Navier-Stokes equation is solved numerically based on the SIMPLE algorithm [11]. SIMPLE algorithm strategy is 
to divide the equation into two equations, namely advectiondiffusion equation and Poisson equation.

$$
\frac{\mathbf{u}_{i, j}^{n+1}-\mathbf{u}_{i, j}^{n}}{\triangle t}=-\nabla \cdot \mathbf{u u}-\nabla \mathbf{p}+\frac{1}{R e} \nabla^{2} \mathbf{u}
$$

Equation (1) is discretized by using forward difference scheme of time variables. Furthermore the Equation (3) is separated by giving new variables that is temporary velocity, and by ignoring the pressure component, the advection equation is obtained as follows:

$$
\frac{\mathbf{u}_{i, j}^{\prime}-\mathbf{u}_{i, j}^{n}}{\triangle t}=-\nabla \cdot \mathbf{u u}+\frac{1}{R e} \nabla^{2} \mathbf{u}
$$

The second equation is formed based on the temporary velocity and pressure components obtained as follows

$$
\frac{\mathbf{u}_{i, j}^{n+1}-\mathbf{u}_{i, j}^{n}}{\Delta t}=-\nabla \mathbf{p}
$$

By adding the gradient operator in Equation (5), the Poisson equation is obtained as follows

$$
\frac{1}{\triangle t} \nabla \mathbf{u}^{\prime}=\nabla^{2} \mathbf{p}
$$

The next step is to update the velocity component with the following equation

$$
\nabla \mathbf{u}=-\nabla \mathbf{p}
$$

The velocity component is updated until the desired condition is obtained. Then velocity and pressure component could be visualized as fluid flow profile.

\section{Computation Domain and Boundary Condition}

The simulation area domain is $20 D \times 10 D$, where $D$ is the diameter of the circular cylinder. Circular cylinders are placed in $(4 D, 5 D)$. First passive control is placed in front of a circular cylinder at distance $S=2.4 D$ from the center of the circular cylinder and two other passive controls are placed behind the circular cylinder at varying distances, namely $1.6 D$ and $1.8 D$. The angle between two passive controls also varies, $\alpha=30^{\circ}, 60^{\circ}, 90^{\circ}, 120^{\circ}$. The simulation domain scheme is shown in Fig. 1. At distance $6 D, 8.5 D$ and $11 D$, we identify the velocity profile in wake. The domain area is formed into $800 \times 400$ grid size. The grid used in discretization is a uniform grid, where the $\triangle x$ and $\triangle y$ are the same. Discretization of equations is done by applying the difference scheme.

$$
\begin{gathered}
\left(f_{x}\right)=\frac{2 f_{i+1, j}+3 f_{i, j}-6 f_{i-1, j}+f_{i-2, j}}{6 d x} \\
\left(f_{y}\right)=\frac{2 f_{i, j+1}+3 f_{i, j}-6 f_{i, j-1}+f_{i, j-2}}{6 d y} \\
\left(f_{x x}\right)=\frac{f_{i+1, j}-2 f_{i, j}+f_{i-1, j}}{d x^{2}} \\
\left(f_{y y}\right)=\frac{f_{i, j+1}-2 f_{i, j}+f_{i, j-1}}{d x^{2}}
\end{gathered}
$$

The fluid flow in the area is assumed to be a uniform flow. So, the velocity values in the free area are $\mathbf{u}_{\infty}=1$ and $\mathbf{v}_{\infty}=0$. The flow is assumed that no friction is received by the fluid flow. When the fluid flow moves continuously without barrier, $\mathbf{u}_{i, n}=\mathbf{u}_{i, n-1}$. The velocity of fluid flow is assumed by $\mathbf{u}=\mathbf{v}=$ 0 .

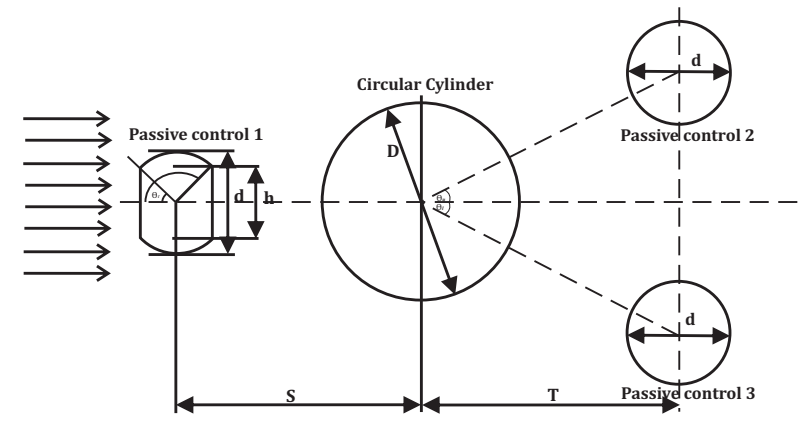

Fig. 1: Simulation scheme for fluid flow around circular cylinder and three passive controls.

\section{Simulation Result}

The results of numerical simulations of Navier-Stoke equations based on the SIMPLE algorithm are obtained by component values of velocity and pressure of fluid flow. Various configurations affect the streamline and pressure distribution around the main cylinder.

\section{A. Streamline Profiles}

Fluid flow around a circular cylinder without passive control can cause vortex behind the circular cylinder irregularly. The vortex that occurs behind the upper cylinder is not symmetrical with the vortex occurring behind the upper cylinder. The vortex occurring behind the circular cylinder without passive control is shown in Fig. 2. Fluid flow through circular cylinders with

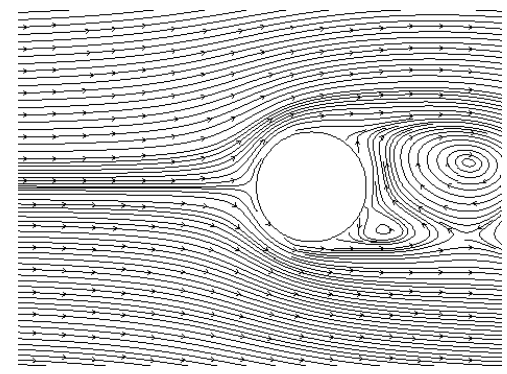

Fig. 2: Streamline profile of flow around circular cylinder without passive control.

passive control has streamline profile difference when compared to circular cylinders without passive control. This can be seen based on the vortex formed. By giving passive control, the formed vortex is more symmetric between the vortex behind the top of the cylinder and the bottom of the cylinder. The position of the passive control placed behind the cylinder also affects the vortex that is formed. Although symmetrical vortices are formed, the size of the vortex produced varies.

Passive control placed behind the cylinder at distance $T=$ $1.6 \mathrm{D}$ is shown at Fig. 3, with a large angle of $30^{\circ}$ between the passive control of the center of the cylinder. The vortex that occurs is quite large and looked asymmetric, where one 


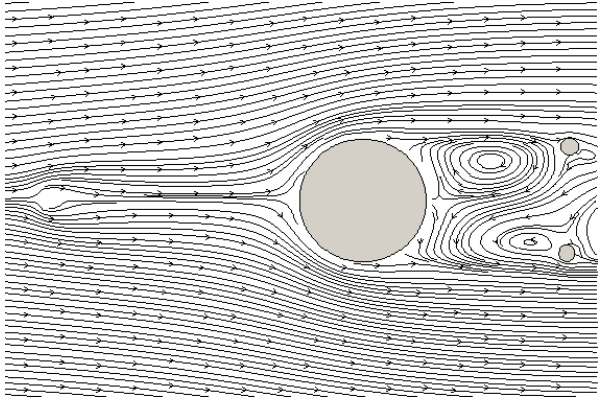

Fig. 3a: Streamline Profile at $\mathrm{T}=1.6 \mathrm{D}$ and $\alpha=30^{\circ}$

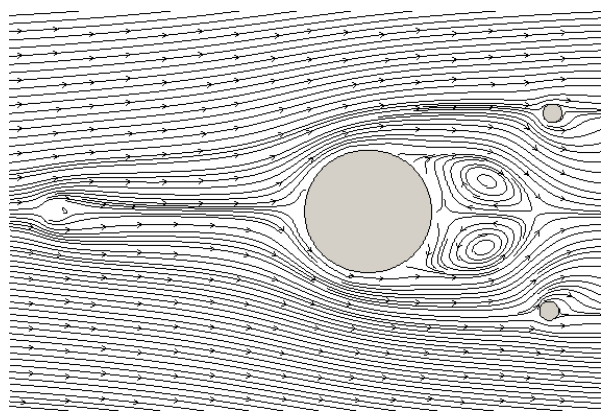

Fig. 3b: Streamline Profile at T=1.6D and $\alpha=60^{\circ}$

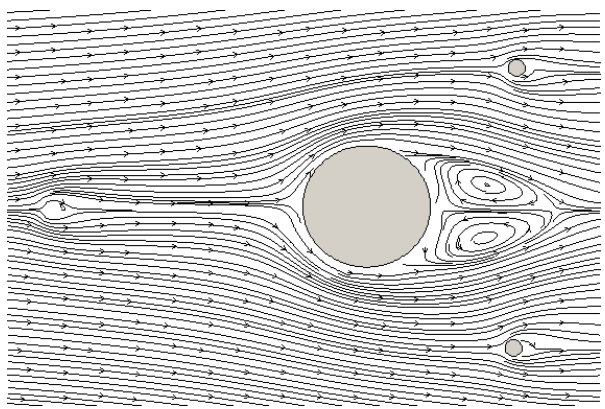

Fig. 3c: Streamline Profile at $\mathrm{T}=1.6 \mathrm{D}$ and $\alpha=90^{\circ}$

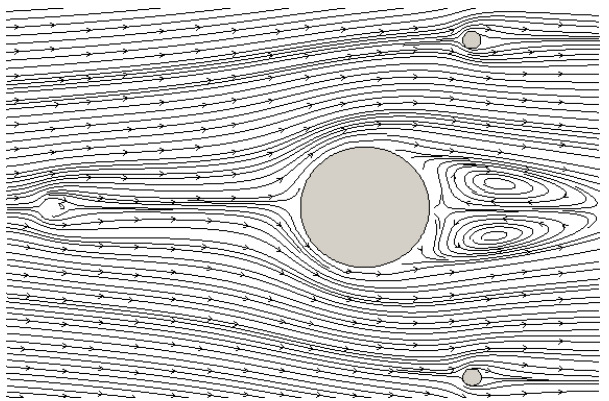

Fig. 3d: Streamline Profile at $\mathrm{T}=1.6 \mathrm{D}$ and $\alpha=120^{\circ}$

vortex occurs at the bottom of the cylinder backwards. Then, at $\alpha=60^{\circ}$, the vortex becomes smaller and more symmetric. At $\alpha>60^{\circ}$, the vortex area becomes larger. Then, when $\alpha<60^{\circ}$ also does not guarantee the vortex area becomes smaller. So, in this experiment, the best $\alpha$ is $60^{\circ}$.

The vortex profile that occurs by placing two passive controls behind the cylinder at distance $T=1.8 D$ is almost the same as at the distance $T=1.6 D$. Streamline profile at

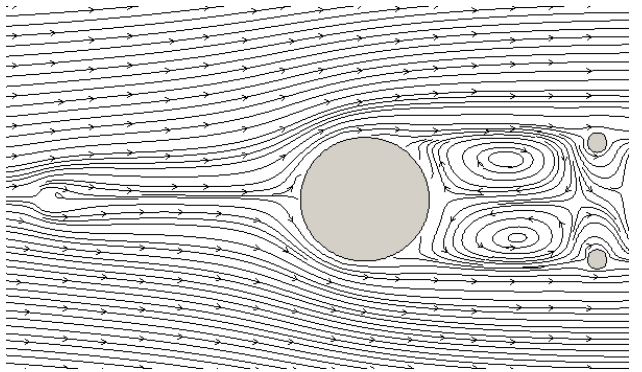

Fig. 4a: Streamline Profile at $T=1.8 D$ and $\alpha=30^{\circ}$

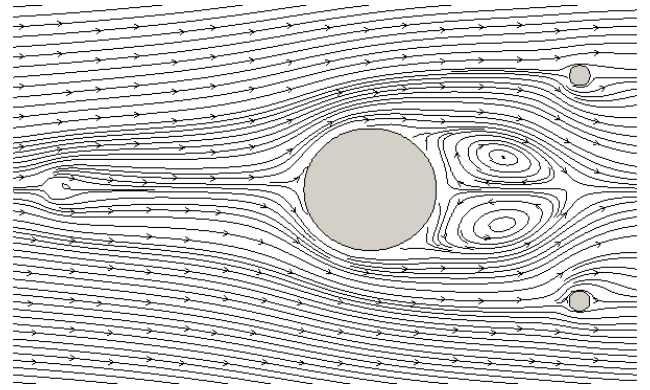

Fig. 4b: Streamline Profile at $T=1.8 D$ and $\alpha=60^{\circ}$

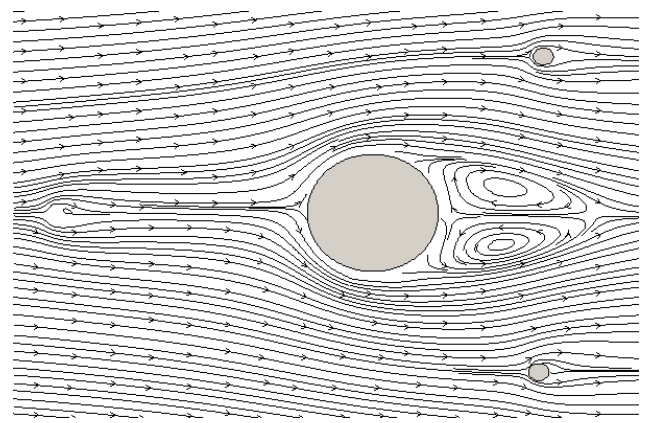

Fig. 4c: Streamline Profile at $T=1.8 D$ and $\alpha=90^{\circ}$

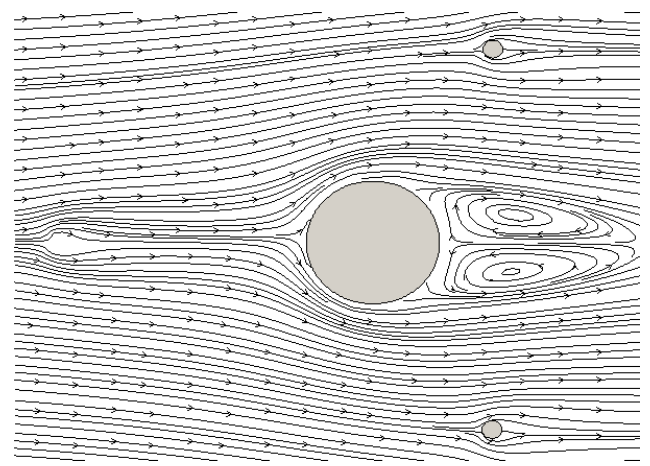

Fig. 4d: Streamline Profile at $T=1.8 D$ and $\alpha=120^{\circ}$

$T=1.8 D$ is shown at Fig. 4.

\section{B. Velocity Profiles in Wake}

The differences that occur by giving passive control behind the cylinder can be considered through the velocity profile that occurs in the wake area. The velocity profile in the wake area is shown in Fig. 5 and Fig. 6. 


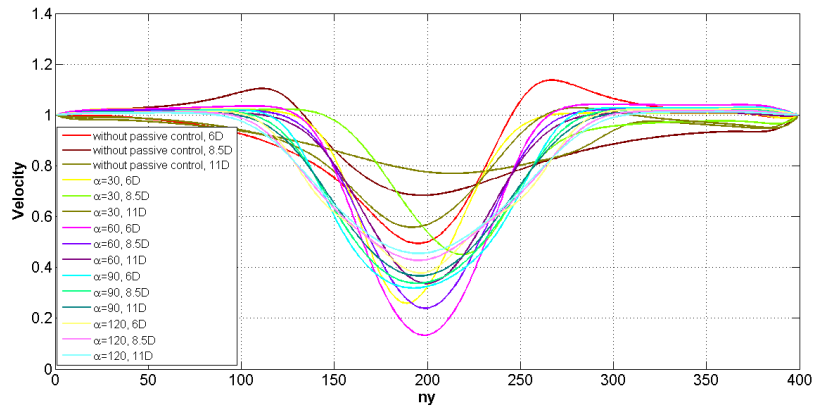

Fig. 5: Velocity Profile at Wake Area When $T=1.6 D$

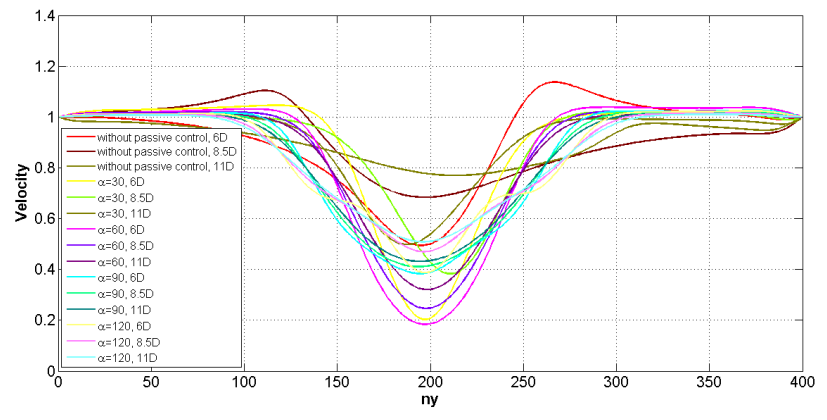

Fig. 6: Velocity Profile at Wake Area When $T=1.8 D$

The velocity profile in the wake area is taken at a distance of $6 D, 8.5 \mathrm{D}$ and $11 \mathrm{D}$ from the center of the circular cylinder. The farther from the center of the circular cylinder, the velocity of the fluid flow is higher. The fluid flow profile behind the circular cylinder without passive control looks more asymmetric between the fluid flow through the top of the cylinder and the bottom of the cylinder. By giving passive control around the circular cylinder, the fluid flow velocity profile that flows from the bottom of the cylinder looks symmetric with the flow of fluid flowing from the top of the cylinder. The decrease in fluid flow velocity occurs after passive control around the circular cylinder. Passive control placed in a position or angle between different passive controls has a different effect. The greatest decrease in fluid flow velocity occurs when two passive controls are placed at a distance of $1.6 \mathrm{D}$ behind the cylinder and the angle between the two passive controls is $60^{\circ}$. The biggest velocity reduction results also occur in the condition that two passive controls are placed behind the cylinder at a distance of $T=1.8 D$ and the large angle between the two passive controls is $60^{\circ}$.

\section{Drag Coefficient}

The drag coefficient is calculated based on the distribution of the pressure coefficient received around the circular cylinder. The formula for calculating the non-dimensional magnitude of the drag coefficient [12] is

$$
c_{d}=\int_{0}^{2 \pi}\left(c_{p} \cos \theta\right) d \theta
$$

where $c_{p}$ is the pressure coefficient that depends on $\theta$. Variable $\theta$ is the angle that determines the position of the pressure
TABLE I: Drag coefficient received by circular cylinder.

\begin{tabular}{c||c|c}
\hline $\begin{array}{c}\text { Reynolds } \\
\text { Number }\end{array}$ & $\begin{array}{c}\text { Passive Control } \\
\text { Position }\end{array}$ & $\begin{array}{c}\text { Drag } \\
\text { Coefficient }\end{array}$ \\
\hline \hline 500 & Without Passive Control (Present) & 1.2338 \\
& Without Passive Control (4) & 1.2329 \\
& Single Passive Control & 0.9220 \\
& $T=1.6 D, \alpha=30^{\circ}$ & 1.0281 \\
& $T=1.6 D, \alpha=60^{\circ}$ & 0.9721 \\
& $T=1.6 D, \alpha=90^{\circ}$ & 0.9882 \\
& $T=1.6 D, \alpha=120^{\circ}$ & 1.0208 \\
& $T=1.8 D, \alpha=30^{\circ}$ & 1.0128 \\
& $T=1.8 D, \alpha=60^{\circ}$ & 0.9627 \\
& $T=1.8 D, \alpha=90^{\circ}$ & 1.0087 \\
& $T=1.8 D, \alpha=120^{\circ}$ & 1.0706 \\
\hline
\end{tabular}

point. The distribution of the pressure coefficient received by the circular cylinder is shown in (7). The biggest pressure received by the circular cylinder is when the angle position is $0^{\circ}$ or at the point of stagnation. Then the pressure drops to the lowest position which is at an angle of $80^{\circ}$. After the pressure in the lowest condition, then it rises again to position $180^{\circ}$. The pressure distribution occurs at the bottom circular cylinder (area that occurs at an angle of $0^{\circ}-180^{\circ}$ ) symmetrical with the pressure distribution received upper circular cylinder (area that occurs at an angle of $180^{\circ}-360^{\circ}$ ). Pressure distribution is shown in Fig. 7. Based on the pressure distribution data

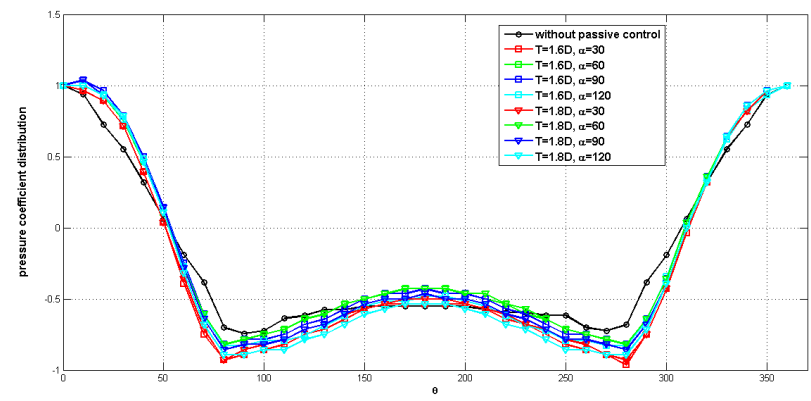

Fig. 7: Distribution of Pressure Coefficient

above, the drag coefficient is calculated using (8). Equations are solved numerically using Simpson rules [13]. The result of the drag coefficient received by the circular cylinder is shown in Table I.

The drag coefficient received by a circular cylinder without passive control is 1.2338. This result is almost the same as the results obtained in [5]. The smallest drag coefficient occurs when two passive controls are placed behind the circular cylinder at a distance of $T=1.6 \mathrm{D}$ and the angle between the two passive controls is $30^{\circ}$. By this configuration, the drag coefficient can be reduced to $21.2109 \%$. However, the decrease of drag coefficient that has been obtained is not smaller than the circular cylinder with a single passive control I-shape cylinder which reaches $25.271 \%$. 


\section{Conclusions}

Based on the simulation results, passive control around the circular cylinder can give effect to the vortex form. Vortex area can be formed to be smaller and symmetry. In addition, the drag coefficient received by circular cylinders can be reduced to $21.2109 \%$. This occurs when a passive control is placed in front of a circular cylinder at the distance of $S=2.4 \mathrm{D}$ and two other passive controls are placed behind the circular cylinder at distances $T=1.6 D$ and $\alpha=60^{\circ}$. However, three passive controls do not produce better results than the passive twocontrol configuration. Thus, it is necessary to modify other passive controls so that it can be more efficient to reduce the drag coefficient received by the circular cylinder.

\section{REFERENCES}

[1] Y. Triyogi, D. Suprayogi, and E. Spirda, "Reducing the drag on a circular cylinder by upstream installation of an I-type bluff body as passive control," Proceedings of the Institution of Mechanical Engineers, Part C: Journal of Mechanical Engineering Science, vol. 223, no. 10, pp. 2291-2296, 2009.

[2] T. Igarashi and Y. Shiba, "Drag reduction for D-shape and I-shape cylinders," JSME International Journal Series B Fluids and Thermal Engineering, vol. 49, no. 4, pp. 1036-1042, 2006.

[3] T. Yuwono and W. Widodo, "Flow characteristic around a D-type cylinder near a plane wall," in Regional Conferences on Mechanical and Aerospace Technology, Bali, 2010.

[4] B. Widodo, T. Yuwono, and C. Imron, "The influence of distance between passive control and circular cylinder on wake," in Journal of Physics: Conference Series, vol. 890, no. 1, 2017, p. 012053.

[5] C. Imron, L. Mardianto, B. Widodo, and T. Yuwono, "Mathematical modeling of drag coefficient reduction in circular cylinder using two passive controls at $\mathrm{Re}=1000$," Mathematical and Computational Applications, vol. 23, no. 1, p. 2, 2018.

[6] A. Hakam, B. Widodo, T. Yuwono, and C. Imron, "Numerical investigation of the flow around circular cylinder with two passive controls," in Journal of Physics: Conference Series, vol. 974, no. 1, 2018, p. 012011.

[7] C.-H. Kuo and C.-C. Chen, "Passive control of wake flow by two small control cylinders at Reynolds number 80," Journal of fluids and structures, vol. 25, no. 6, pp. 1021-1028, 2009.

[8] Y. Bao and J. Tao, "The passive control of wake flow behind a circular cylinder by parallel dual plates," Journal of Fluids and Structures, vol. 37, pp. 201-219, 2013.

[9] L. Lu, M. Liu, B. Teng, Z. Cui, G. Tang, M. Zhao, and L. Cheng, "Numerical investigation of fluid flow past circular cylinder with multiple control rods at low Reynolds number," Journal of Fluids and Structures, vol. 48, pp. 235-259, 2014.

[10] H. Versteeg and W. Malalasekera, An introduction to computational fluid dynamics: the finite volume method. Pearson education, 2007.

[11] M. Matyka, "Solution to two-dimensional incompressible Navier-Stokes equations with simple, simpler and vorticity-stream function approaches. driven-lid cavity problem: Solution and visualization," arXiv preprint physics/0407002, 2004.

[12] T. Bergman, F. Incropera, D. DeWitt, and A. Lavine, Fundamentals of heat and mass transfer. John Wiley \& Sons, 2011.

[13] R. Burden and J. Faires, "Numerical differentiation \& integration numerical differentiation I," Numerical analysis, pp. 174-184, 2011. 